\title{
Effect of the chute oscillations frequency on the speed of inertial-conveyor transportation of concrete mix
}

\author{
Sergey G. Osmanov ${ }^{1, *}$ \\ ${ }^{1}$ Don State Technical University (DSTU), Department of Building Technology, 344022 Rostov-on-Don, \\ Russia
}

\begin{abstract}
The paper represents the major advantages of use of the author's technology of concrete mix feeding to the point of placement in a building site environment. The working procedure of the experimental research of the effect of frequency work regimes of involved in this case inertia conveyors with the working body in the form of a chute on the transportation speed of ready-mix concrete on the dense aggregate is described and proved. The results of this research are given, particularly, it is shown how an optimal frequency of chute oscillations depends on the angle of its slope and technological properties of transported mix.
\end{abstract}

\section{Introduction}

In a building site environment transportation of ready-mix concrete and, namely, its feeding to the point of placement, as has been mentioned by several researchers [1-3], is considered to be one of the most resource-intensive components of the complex engineering process of building structures concreting. The application of vibrating conveyors as the means of such transportation, among others together with the hoisting cranes, in a number of cases, for example while constructing foundations [4], hydraulic structures [5], and also in the process of concrete works execution inside the buildings under construction and reconstruction [6], gives a chance to reach a sizable saving of material and technical, labor and energy resources in comparison with the other possible mechanization variants.

The working body of such conveyors is an oscillating chute, due to what a considerable thickness of the mix layer transported with them and, as a consequence, their high capacity, can be provided. As the concrete mix inertia forces are the only forces, aimed it at set in motion to the point of placement at any chute slope angles $(\alpha)$, the vibrating conveyors should be regarded as [7] the kind of inertial ones. The latter, as it is known, differ by the simplicity of construct and maintenance, low material consumption and high operational dependability [8].

However, even the most successful standard modifications of such devices (so called "vibrating chutes"), equipped with the circular harmonic oscillators with the amplitude $(A)$

* Corresponding author: osmanov.tsp@ya.ru 
and the frequency $(f)$, accordingly amounting to $0.2-0.6 \mathrm{~mm}$ and $2800 \mathrm{~min}^{-1}$, nevertheless, have no widespread application in practice. It is connected with considerable limits, among others, both by the characteristics of concrete mix workability, effectively transported at predetermined values of $\alpha$, and by the distance of such transportation, since the paths of these conveyors must be laid with the downward gradient at $\alpha \geq+5^{\circ}$ [9].

The technology of concrete mix feeding to the point of placement [10], proposed and patented by the author, renders possible the transportation of the mixes of any slump and Vebe classes (according to the Russian State Standard 7473-2010) with normative and higher capacity of the inertia conveyor not only at the slope of chute downward, but also horizontally (i.e. at $\alpha=0^{\circ}$ ) and its some upward slope (e.g. up to $-10^{\circ}$ for the mixes with slump of $15 \mathrm{~cm}$ ). This, in turn, allows to feed the mix at greater distances than before and with lower inputs of material and technical resources and labor for the laying and maintenance of conveyor paths [11]. Thus, the author could expand considerably the spectrum of the conditions of possible effective application of inertia conveyors in structures concreting. The achieved intensification of the transportation process is determined, mainly, by its implementation in low-frequency regimes of longitudinal asymmetric oscillations with a high amplitude, which are divided by means of short-time pauses and come into fruition due to cam mechanism in a drive unit of the conveyor plant. The energy of such oscillations is expended principally not for the fluidization of mix, as before $[12,13]$, but for its shift in disharge direction that in a concept plan became a distinguishing feature of the proposed approach.

It is ascertained that the increase of chute oscillations frequency, inevitably entailing the increase of mix inertia forces, and consequently, dynamic loads on the conveyor construct, also leads, at first, to the rise and afterwards the stabilization and drop of the transportation speed (v). Meanwhile, this happens with the rates depending on mix workability and predetermined values of $\alpha$. Therefore, it is highly important to set the most expedient (optimal) values of $f$, after the achievement of which further rise of this speed (in contrast to the above mentioned loads) either would be absent or of little significance. In practice it could allow to provide not only high technical and economic indicators of concrete mix transportation process, but its highest energy efficiency.

\section{Experimental programme}

It is necessary to note that the ascertainment of optimal pattern of the chute oscillation asymmetry and the values defining its parameters preceded the solution to the marked above objective. Firstly, by means of this it became possible to activate so called "latent" reserves of the transportation speed raise [14], the use of which does not mean the significant increase of conveyor plant energy consumption. Secondly, the procedures mentioned allowed to provide the possibility of existence and afterwards the detection of less (in average by $10 \%$ ) optimal values of $f$, corresponding, as a consequence, to less unit inputs of energy on mix transportation.

In experiments on the detection of optimal frequency regimes of transportation concrete mixes of 1:2.25:3.25 (C:S:R) composition on CEM I 42.5 N Portland cement (ZAO Oskolcement), granite rubble of 5-20 mm size (OAO Pavlovskgranit) and silica sand with fineness modulus of 2.1 from the Don river were used. The range of $W / C$ in tested low, medium and high workable (with slump of 1 to $25 \mathrm{~cm}$ ) and stiff consistency (with Vebe time of 20 to $100 \mathrm{~s}$ ) mixes amounted in total to $0.35-0.74$. The mix with current workability, assigned in increments of $5 \mathrm{~cm}(20 \mathrm{~s})$, was transported by means of experimental inertia conveyor (Figure 1a) with $3 \mathrm{~m}$ length polypropylene chute at the ascertained before corresponding optimal values of parameters of oscillations asymmetry and their predetermined amplitude $(25,35,55$ и $85 \mathrm{~mm})$. Simultaneously, for a further 
transportation speed determination, successively at each experimental value of $\alpha(+10,+5$, $0,-5,-10$ and $-15^{\circ}$ ) a series of records of output information was conducted. The latter were performed in increments of $\Delta f=15 \mathrm{~min}^{-1}$ that was monitored with SK-T2 clock-type tachometer. The chute diameter and the maximal thickness of transported mix layer amounted to $150 \mathrm{~mm}$ and $10 \mathrm{~cm}$ respectively.

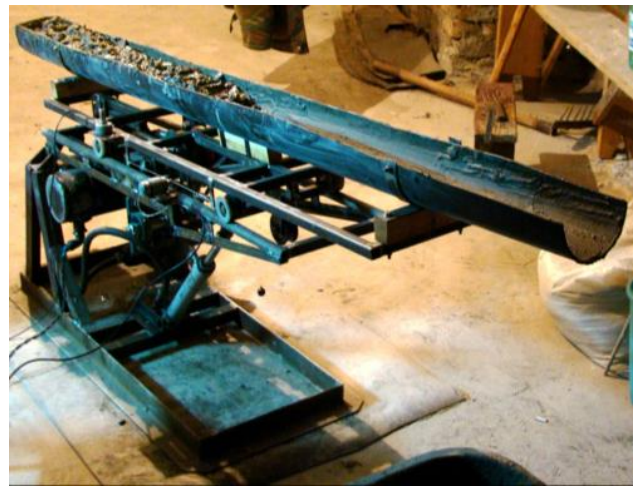

a)

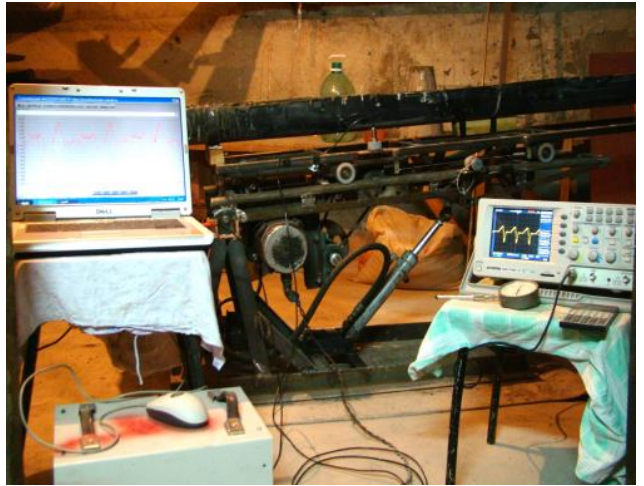

b)

Fig. 1. Experimental inertia conveyor: general view (a) and side view in the process of taking of oscilloscope readings for actual $\mathbf{V}_{\max }$ values determination (b).

Recorded information, among other things, included the data about transit time by the tagged particle, placed on the surface of the moving mix, the distance $(50 \mathrm{~cm})$ between two Omron E3T series photoelectric sensors designed to trigger on diffuse reflection of light. They, in turn, were connected to GW Instek GDS-71022 laptop-compatible digital oscilloscope, which operated in the regime of recorder. The tagged particle was the foampolystyrene ball with the diameter of $10 \mathrm{~mm}$, coated with retro-reflective enamel. Precise values of $\boldsymbol{v}$ were determined by means of corresponding oscillograms files processing.

As the optimality criterion of frequency regimes the achievement of the maximum of $\Psi$ (coefficient of velocity transfer from the chute $(\mathbf{V})$ to the concrete mix) was taken:

$$
\Psi \rightarrow \Psi_{\max }=\max \left(\mathbf{v} / \mathbf{V}_{\max }\right) .
$$

This testified to the equality of the decreasing and constant rise rates of the transportation speed and the oscillations frequency respectively. In this regard, it is important to note that at $\Psi>\Psi_{\max }$ the second of these rates is higher than the first one. Therefore, a further increase in the frequency would lead not so much to the intensification of the engineering process under study, but to an intensive increase in the inertia forces in the construct of the conveyor plant and, as a consequence, the emergence of rather high dynamic loads on it. It goes without saying that the corresponding rise of $\boldsymbol{v}$ values, in this case, would be considered not only of little significance, but also ineffective.

In the course of condition (1) filtering of the experiments results the values of $\mathbf{V}_{\max }$ were calculated by the following formula:

$$
\mathbf{V}_{\max }=2 \pi f \mathbf{U}_{\max }
$$

where $\mathbf{U}_{\max }$ is the maximum of the chute velocity analogue determined from the equations of the piecewise function $\mathbf{U}(\varphi)$. The latter were derived earlier by integration of the corresponding function of the acceleration analogue [10]. The results of $\Psi$ coefficient calculation (to control the 
closure of the highest pair and, as a consequence, the adherence to the accepted modified sinusoidal law of chute motion) were also tested by means of the actual $\mathbf{V}_{\max }$ values. These values, in turn, were found by a special advance prepared scale. With its help the conversion of the recorded (Figure $1 \mathrm{~b}$ ) on oscillograms peaks of the voltage of DC produced by the chuteconnected universal transducer (tachogenerator) into the chute velocity was executed.

\section{Results and discussion}

On the basis of many experimentally obtained "working" charts, displaying the effect of frequency regimes on the transportation speed, "objective" charts, showing how the optimal frequency $\left(f_{\Psi}\right)$ depends on assigned oscillations amplitude, were generated. Examples of the first and second charts are presented in Figure 2 (the achievement of $\Psi_{\max }$ is denoted with markers in the form of asterisks) and Figure 3 respectively. It is interesting that common range of $f_{\Psi}$ for both low, medium and high workable and stiff consistency mixes appeared to be approximately identical and amounted to $90-255 \mathrm{~min}^{-1}$.

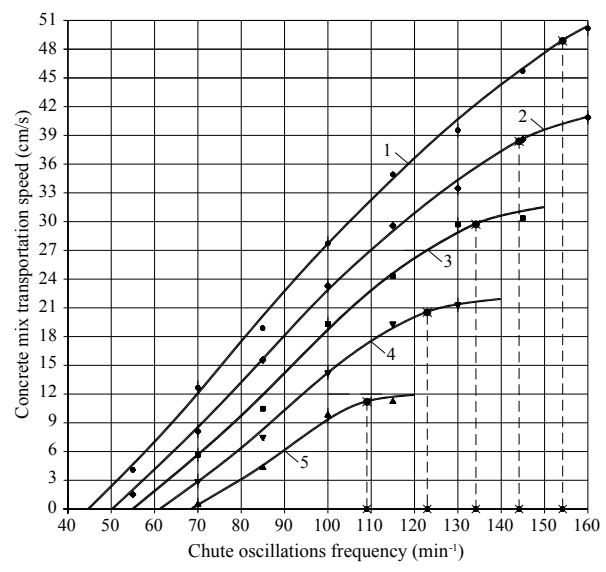

a)

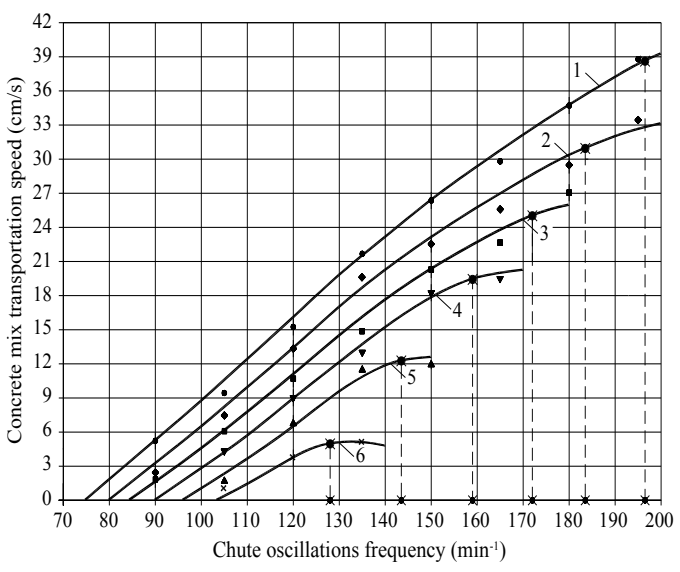

b)

Fig. 2. Dependence of speed of transportation of mixes with slump of $15 \mathrm{~cm} \mathrm{(a)} \mathrm{and} \mathrm{Vebe} \mathrm{time} \mathrm{of}$ $60 \mathrm{~s} \mathrm{(b)} \mathrm{on} \mathrm{chute} \mathrm{oscillations} \mathrm{frequency} \mathrm{at} A=55 \mathrm{~mm}$ and $\alpha$ equal to $+10(1),+5$ (2), 0 (3), -5 (4), -10 (5) and $-15^{\circ}(6)$.

Detailed block-by-block analysis of the materials of video filming of moving in the chute mix allowed us to state that its maximal (critical) forward acceleration, directed towards discharge $\left(\tilde{\mathbf{a}}_{\mathbf{f}, \max }\right)$, can be identified with the acceleration, taking place at condition (1) observance. The excess of $\tilde{\mathbf{a}}_{\mathbf{f}, \max }$ values is accompanied by the loss of relative uniformity and stability of the mix movement as a continuous flow. Mathematical treatment of data obtained from the experiments results made it possible to ascertain this acceleration. Besides, a special interest is that found values of $\tilde{\mathbf{a}}_{\mathbf{f}, \max }$ (Table 1) are also constant in relation to chute oscillations parameters and therefore, per se, complete the available knowledge about technological properties of normal weight concrete mixes.

As it appeared to be afterwards, critical acceleration of the chute can be used to calculate its optimal frequency of oscillations at any initially predetermined amplitude by the following formula analytically obtained by the author:

$$
f_{\Psi}=0.5 \pi^{-1.5}\left(\Phi_{\text {opt. }} A^{-1} \tilde{\mathbf{a}}_{\mathbf{f}, \max }\right)^{0.5},
$$


where $\Phi_{\text {opt. }}$ is the optimal (for existing production conditions) value of generalized asymmetry parameter depending on corresponding set of particular asymmetry parameters [14]. Convergence of the results of calculations by the formula (3) with experimental data (Figure 3) amounts to about $95 \%$.

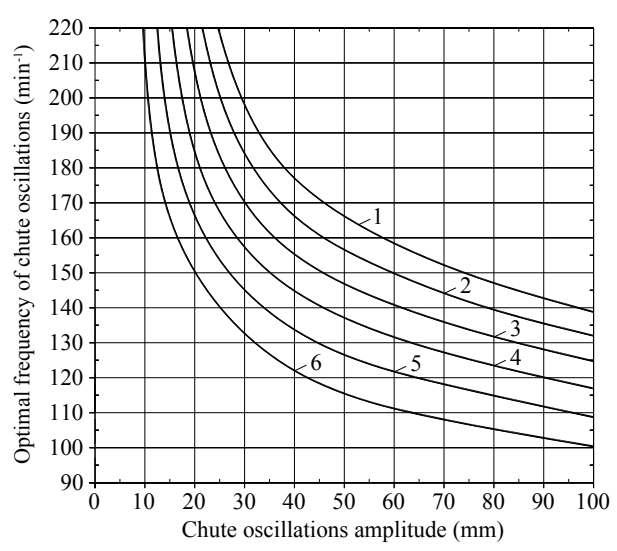

a)

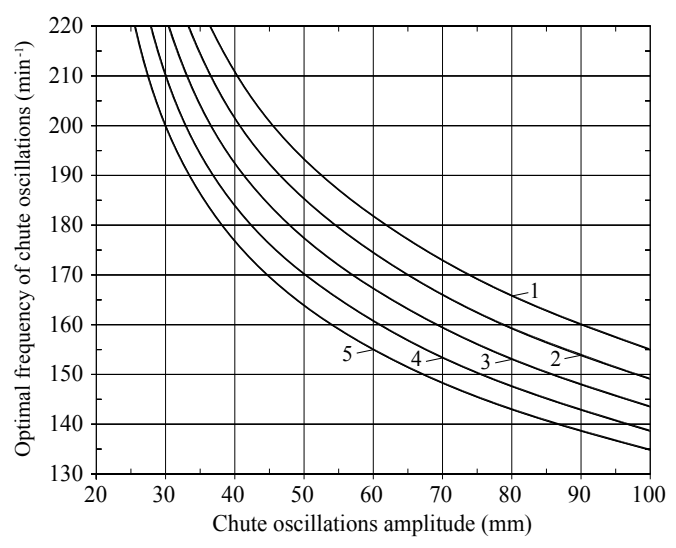

b)

Fig. 3. Dependence of chute oscillations optimal frequency on their amplitude at horizontal transportation of: low, medium and high workable mixes (a) with slump equal to 1 (1), 5 (2), 10 (3), 15 (4), 20 (5) and $25 \mathrm{~cm}$ (6); stiff consistency mixes (b) with Vebe time equal to 20 (1), 40 (2), 60 (3), 80 (4) and $100 \mathrm{~s}(5)$.

Table 1. Conditions of effective transportation of low, medium and high workable and stiff consistency mixes.

\begin{tabular}{|c|c|c|c|c|c|c|c|c|c|c|c|}
\hline \multirow{3}{*}{$\begin{array}{l}\text { Chute } \\
\text { slope } \\
\text { angle } \\
\text { (deg) }\end{array}$} & \multicolumn{11}{|c|}{ Critical chute acceleration $\left(\mathrm{m} / \mathrm{s}^{2}\right)$ at the characteristics of concrete mix workability } \\
\hline & \multicolumn{6}{|c|}{ Slump (cm) } & \multicolumn{5}{|c|}{ Vebe time (s) } \\
\hline & 1 & 5 & 10 & 15 & 20 & 25 & 20 & 40 & 60 & 80 & 100 \\
\hline+10 & 19.28 & 16.71 & 14.42 & 12.29 & 10.61 & 8.86 & 26.14 & 24.65 & 23.30 & 22.58 & 22.26 \\
\hline+5 & 16.72 & 14.58 & 12.64 & 10.77 & 9.15 & 7.64 & 22.70 & 21.17 & 20.08 & 19.28 & 18.83 \\
\hline 0 & 14.09 & 12.08 & 10.38 & 8.90 & 7.44 & 6.14 & 18.99 & 17.76 & 16.66 & 15.75 & 15.26 \\
\hline-5 & 11.30 & 9.41 & 7.52 & 6.00 & 4.82 & 3.71 & 15.77 & 14.42 & 13.55 & 12.73 & 12.18 \\
\hline-10 & 8.54 & 6.94 & 5.30 & 4.09 & - & - & 12.89 & 11.74 & 10.93 & 10.25 & 9.75 \\
\hline-15 & 6.37 & 5.06 & - & - & - & - & 10.41 & 9.44 & 8.69 & 8.07 & 7.59 \\
\hline
\end{tabular}

Note: impossibility of stable transportation of mix with inertia conveyor is denoted by "-"

The stable (see the note to Table 1) transportation of concrete mix according to assumption made by the author is characterized by the achievement of $v \geq 3,5 \mathrm{~cm} / \mathrm{s}$. This is the minimum that, in principle, may have a practical interest at the design of the process of structures concreting. Maximal slope angle of the chute upward corresponding to the present value of $\boldsymbol{v}$ largely depends on oscillations amplitude. It is ascertained that at the optimal values of their frequency this angle can reach up to $-20^{\circ}$ in the case of transportation of stiff consistency mixes (virtually unaffected by Vebe time) and up to $-15^{\circ}$ (depending on slump) in transportation of low, medium and high workable mixes. 


\section{Conclusions}

While evaluating results of the presented research in general it is necessary to note that inertial-conveyor transportation of concrete mixes in detected optimal frequency regimes happens with the speeds on average up to 2-6 times higher than at the use of different vibrating conveyors. Exactly the same in this case the capacity of the latter is lower as compared to the inertia conveyors constructed according to the author's patent. These circumstances, taking into account the proven [15] absence of negative influence of the author's technology on the physical and mechanical properties of concrete in structures, allow us to recommend this technology for application in construction production.

\section{References}

1. Yu.G. Khayutin, Monolithic concrete: technology of works execution (Stroyizdat, Moscow, 1991)

2. B.V. Zhadanovsky, Mechanization of construction, 11, 21-25 (2003)

3. S.G. Osmanov, Proceedings of Rostov State University of Civil Engineering, 15, 368369 (2011)

4. L.S. Rozenboym, Small-scale mechanization of concrete works (Stroyizdat, Moscow, 1984)

5. P.S. Neporozhniy, Constructing of large concrete and reinforced concrete hydraulic structures (Gosstroyizdat USSR, Kiev, 1958)

6. A.A. Afanas'ev, Constructing of buildings and engineering structures made of monolithic reinforced concrete (Stroyizdat, Moscow, 1990)

7. R.L. Zenkov, I.I. Ivashkov, L.N. Kolobov, Machines of continuous transport (Mashinostroenie, Moscow, 1987)

8. A.O. Spivakovskiy, V.K. D"yachkov, Transportation machines (Mashinostroenie, Moscow, 1983)

9. I.G. Sovalov, Ya.G. Mogilevskiy, Mechanization of concrete works while constructing monolithic structures (Stroyizdat, Moscow, 1977)

10. S.G. Osmanov, A.L. Zholobov, Bulletin of Civil Engineers, 4, 138-145 (2012)

11. S.G. Osmanov, Science Review, 10.2, 370-374 (2014)

12. I.G. Sovalov, I.A. Shenderovich, Concrete and reinforced concrete, 5, 19-21 (1967)

13. B.A. Lishansky, N.V. Mikhailov, Concrete and reinforced concrete, 3, 35-37 (1970)

14. S.G. Osmanov, VIth International scientific-practical conference "Building sector development prospects", 2, 3-9 (Astrakhan Institute of Civil Engineering, Astrakhan, 2012)

15. S.G. Osmanov, Solid State Phenomena, 265, 97-102 (2017) 